\title{
Erratum
}

\section{Genomewide analysis of NBS-encoding genes in kiwi fruit (Actinidia chinensis)}

Yingjun Li, Yan Zhong, Kaihui Huang and Zong-Ming Cheng

J. Genet. 95, 997-1001

The following statement in the footnote of the first page is missing in the published version of the above article.

'Yingjun Li and Yan Zhong contributed equally to this work.' 